hopes to use sophisticated software to assemble the enormous jigsaw by matching their sequences to work out which fragment goes where.

Venter has won over many sceptics with his recent completion of the 180-millionbase-pair genome of the fruitfly Drosophila melanogaster (see Nature 401, 729; 1999). But Philip Green, a biocomputing expert at the University of Washington, points out that the human genome is not only much larger - about 70 million fragments will need to be assembled - but also contains many more families of repeat sequences.

The main problem in sequencing the human genome is that, much more than in lower organisms, it contains many repeat sequences of DNA that look identical and are therefore difficult to place. While Dunham's team have managed to resolve all but 11 of the gaps in the 'euchromatic' region of chromosome 22, they stopped short of attempting to unravel the repeat minefield of the 'heterochromatic' regions.

These are not only difficult to sequence, but are full of repeats. This makes it difficult to resolve the sequence stretches on the short ' $p$ ' arm of chromosome 22 from similar repeated heterochromatic regions on chromosomes 21, 13, 14 and 15, for example.

Nonetheless, the success of the clone-byclone approach in resolving repeats in the euchromatic region has galvanized its supporters. Dunham attributes much of this success to the modular design of the cloneby-clone approach. Given a problem such as a region whose depth of coverage is too low - or which contains gaps or repeats - the modular strategy allows researchers to pull the clone for that region from the fridge and do more work on it. It also allows sequencing to be customized to specific regions, such as GC-rich zones, he adds.

Green argues that the clone-by-clone approach is superior to Celera's wholegenome approach as it reduces the scale of the problem, since each clone will have fewer copies of any given repeat. Finishing techniques such as chromosome walking are difficult on a single clone, and sceptics predict that they would be intractable at the level of the whole genome.

Celera's approach to finishing lacks this safety net and its effectiveness in the human genome remains largely unknown. Also, Celera's approach means that it will only be able to attempt finishing late in the day, when it has the entire sequence.

"The self-sufficiency of Celera's wholegenome strategy for the human will never be put to the test," asserts David Page, a genome scientist at the Whitehead Institute at the Massachusetts Institute of Technology. "Celera's proposed sequencing of the human genome will fully exploit and be utterly dependent upon publicly available HGP mapping and sequencing data." Declan Butler

\title{
Tiny chromosome is rich in genes and medical promise
}

London

If all the cell's a stage, and the chromosomes merely players, we still don't know whether chromosome 22 has a bit-part or a leading role. But despite its diminutive size - it is one of the smallest of our 23 pairs of chromosomes - we do know it is likely to be quite gene-rich.

So far, for example, chromosome 22 has been implicated in schizophrenia, chronic myeloid leukaemia and trisomy 22 , the second most common cause of miscarriages. At least 27 diseases involve some genetic component on the chromosome, which is frequently involved in tumour progression.

About half of its genes, however, have yet to be characterized. Their sheer variety, in terms of differing lengths, is of considerable interest. But the relevance of this won't be known until we have more information from other human chromosomes. So which will be sequenced next?

Greg Schuler, group leader of genome resources group at the National Center for Biotechnology Information, says his short list of candidates would include chromosomes 21 and 7, based on the amount of these sequenced so far (see figure).

"The emphasis on the draft map of the human genome has delayed finishing these chromosomes a little, and researchers are
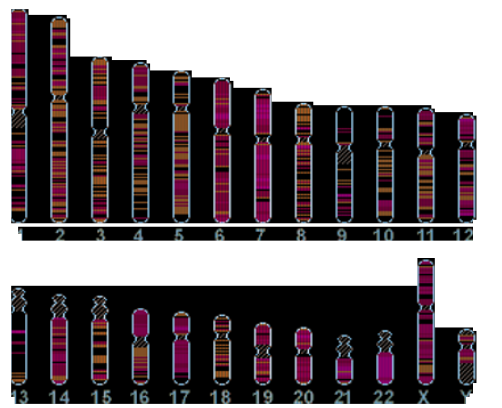

One down, more to go: sequencing of the other chromosomes is progressing at varying rates.

planning for these next year," says Schuler, adding that the Sanger Centre itself is projecting completion of chromosome 20 .

"Things change quite rapidly," Schuler says, so it is difficult to be precise about which will be completed next. The chromosomes are at least partially distributed to different labs, and each of these has its own way of prioritizing.

Completion, says Schuler, is "an accident of how [the labs] decide to queue them up". And what happens when all the chromosomes are complete? "We'll have a big party, of course," he says.

Natasha Loder

\section{Unfinished sequence - the catch on 22}

London

Publication of the sequence of chromosome 22 (see pages 467 and 489) means that, for the first time since the Human Genome Project was proposed in the mid1980s, researchers have an almost continuous sequence for a whole chromosome.

There is, however, a catch. The published sequence remains 'almost continuous' - there are still 11 irritating unsequenced gaps, accounting for about 3 per cent of the sequence. When researchers call the sequence 'complete', they mean they've gone as far as possible with routine methods.

Why do these gaps exist? Researchers on the Human Genome Project sequence DNA by isolating small pieces of chromosome inside bacterial clones or BACs (see previous page) which multiply to produce sequenceable quantities of the DNA segment. But, for various reasons, clones for certain sequences are simply absent from the collection of clones, or 'clone libraries'.

According to lan Dunham of Britain's Sanger Centre, the head of the team that sequenced chromosome 22, most of the gaps contain a sequence that is not stable in Escherichia coli or yeast. One gap does have a corresponding clone; but, for some unknown reason, researchers are unable to sequence it.

As Peter Little, of Imperial College London, puts it, many pieces of DNA "do really weird things when you try to work with them, and we have no idea why".
Based on what he has seen in chromosome 22, Dunham speculates that missing sequences will tend to occur in certain regions of chromosomes: clustered at the ends, for example, with some gaps at the centres involving repeated sequences.

"The good news is that we can now see how big the gaps are," says Dunham: they are between 50 and 150,000 base pairs. If an interesting gene is thought to be in one such gap, many strategies currently being developed could close these gaps.

"It's a matter of fiddling there is no reason to suppose these pieces cannot be done," says Little, adding that it would make very little difference to the overall list of genes on this particular chromosome. 